\title{
Formación profesional. Significados psicológicos desde la perspectiva de género
}

\section{Vocational training. Psychological meanings from the gender perspective}

\author{
YAÑEZ-FLORES, Sara Margarita†*, HERNÁNDEZ-CUETO, Jaquelina Lizet, SALINAS-AGUIRRE, \\ María del Consuelo y VALDES-URIBE, Alejandra Guadalupe
}

Universidad Autónoma de Coahuila-Facultad de Ciencia, Educación y Humanidades, Campus Saltillo

ID $1^{\mathrm{er}}$ Autor: Sara Margarita, Yañez-Flores / ORC ID: 0000-0002-4750-4244, Researcher ID Thomson: S-9231-2018, CVU CONACYT ID: 352125

ID $1^{\text {er }}$ Coautor: Jaquelina Lizet, Hernández-Cueto / ORC ID: 0000-0002- 3728- 7434, Researcher ID Thomson: S-85882018, CVU CONACYT ID: 322702

ID $2^{\text {do }}$ Coautor: María del Consuelo, Salinas-Aguirre / ORC ID: 0000-0002-6542-1813, Researcher ID Thomson: S-92442018, CVU CONACYT ID: 615335

ID $3^{\text {er }}$ Coautor: Alejandra Guadalupe, Valdes-Uribe

DOI: $10.35429 /$ JET.2020.4.11.23.35

Recibido: 25 de Marzo, 2020; Aceptado 19 de Junio, 2020

\section{Resumen}

Las representaciones psicológicas, como reflejo de las creencias, normas y valores, son aderezadas por las perspectivas individuales fruto de sus experiencias y expectativas, que son el resultado de la constante interacción de los estudiantes con su contexto sociocultural lo que permite estudiar, a partir de la palabra estímulo formación profesional, el reflejo de la cultura en las configuraciones cognitivas de los estudiantes. El objetivo de la investigación es analizar las diferencias de significados psicológicos de formación profesional en mujeres y hombres a partir de la carrera estudiada; el diseño de investigación es cualitativa, transversal y muestreo por disponibilidad; los resultados se fundamentan en la perspectiva de 57 estudiantes que evidencia las interacciones a través de los significados psicológicos que mujeres y hombres expresan con la técnica de las Redes Semánticas Naturales (RSN). La contribución del presente estudio es el uso de la técnica de las RSN para identificar los códigos compartidos de los estudiantes que reflejan los significados psicológicos sobre la propia formación profesional.

Formación profesional, Perspectiva de género, Representaciones psicológicas

\begin{abstract}
Psychological representations, as a reflection of beliefs, norms and values, are spiced up by individual perspectives as a result of their experiences and expectations, which are the result of the constant interaction of students with their sociocultural context, which allows studying, based on the palabra estímulo vocational training, the reflection of culture in the cognitive configurations of students. The objective of the research is to analyze the differences in psychological meanings of professional training in women and men from the studied career; the research design is qualitative, cross-sectional and sampling by availability; The results are based on the perspective of 57 students who show the interactions through the psychological meanings that women and men express with the Natural Semantic Networks (RSN) technique. The contribution of the present study is the use of the RSN technique to identify the shared codes of the students that reflect the psychological meanings about their own professional training.
\end{abstract}

Professional training, Gender perspective, Psychological representations

Citación: YAÑEZ-FLORES, Sara Margarita, HERNÁNDEZ-CUETO, Jaquelina Lizet, SALINAS-AGUIRRE, María del Consuelo y VALDES-URIBE, Alejandra Guadalupe. Formación profesional. Significados psicológicos desde la perspectiva de género. Revista Teoría Educativa. 2020. 4-11: 23-35

\footnotetext{
* Correspondencia del Autor (Correo electrónico: sarayanez@uadec.edu.mx)

$\dagger$ Investigador contribuyendo como primer autor.
} 


\section{Introducción}

La información y el conocimiento es una de las armas más valiosas y competitivas de nuestra época. Es atrevido decir que el conocimiento es aún más valioso que una gran cuenta en el banco o una gran fábrica, ya que las empresas que triunfan en el actual mundo globalizado, son aquellas que poseen la mejor información y saben emplearla de una manera eficiente.

Actualmente, dicha afirmación se puede ejemplificar con diversas empresas transnacionales que tienen un éxito rotundo las cuales, lograron su grandeza, no por ser más ricas, sino por poseer algo mucho más valioso, Capital Intelectual.

El concepto de Capital Intelectual ha sido utilizado en la literatura académica desde hace muchos años; es un tópico sobre el cual, el interés de las organizaciones ha crecido rápidamente en los últimos años. En este sentido, Bontis (1998) afirma que "el capital intelectual ha sido considerado por muchos, definido por algunos, entendido por pocos y formalmente valorado por prácticamente nadie", lo cual representa uno de los desafíos más importantes para las empresas actuales.

Bradley (1998), argumenta que el capital intelectual consiste en la capacidad para transformar el conocimiento y los activos intangibles en recursos que crean riqueza tanto en las empresas como en los países. De igual modo, para Edvinsson y Sullivan (1996) y Sullivan $(1999,2001)$ el capital intelectual es aquel conocimiento que puede ser convertido en beneficio en el futuro y que se encuentra formado por recursos tales como las ideas, los inventos, las tecnologías, los programas informáticos, los diseños y los procesos. Tomando como base esta definiciones, se puede concluir que el Capital Intelectual es la suma de conocimientos que poseen todos los colaboradores de una organización y le dan a ésta, una ventaja competitiva.

Para el presente trabajo de investigación se indaga de manera integral en el área académica de una institución de educación media superior, en el estado de Tabasco, y así, ubicar los factores que determinan una influencia determinante, en la generación de capital intelectual, para concluir con el desarrollo de una propuesta de mejora.

\section{Planteamiento del problema}

Las instituciones de educación media superior, al igual que todas las instituciones que se centran en la educación, en el estado de Tabasco enfrentan varios cambios y retos académicos de suma importancia que están siempre enfocados a la mejora continua para brindar una educación de alta calidad a los jóvenes de la región y todos los actores que intervienen en el proceso.

Es por ello, una de las razones por las cuales se desea valorar la incidencia que tiene el capital intelectual dentro de la entidad, porque este es uno de sus activos intangibles más valiosos siendo este un factor fundamental para enfrentar dichos cambios y retos institucionales. Por esta razón es importante implementar una metodología que nos permita valorar el capital intelectual. Es importante que la institución este consciente de que su capital intelectual esté presente en todas las dependencias, y que necesaria y frecuentemente este, está impactando directamente a docentes y estudiantes de los diferentes programas académicos.

En los últimos años, la importancia del capital intelectual dentro de una organización ha ido tomando cada vez más fuerza, y lo que este aporta y representa para la eficiencia de la misma. No obstante hay que tener claridad que las instituciones tanto públicas como privadas, $\mathrm{y}$ sociedad en general carecen totalmente de sistemas de medición que cada vez se hacen más necesarias para estas nuevas fuentes de riqueza, como lo es el objeto de estudio. Por tal motivo, el valorar el capital intelectual y su identificación llegó a ser objeto de estudio para ser medido cuantitativamente de alguna forma.

Lo que se busca, es que la organización pueda administrar con efectividad sus activos inmateriales presentes en cada dependencia de la organización, caso particular los docentes para que estos puedan ser unos proveedores de conocimiento distintivo, aportando a los estudiantes de la institución y fomentar por medio de la enseñanza, la capacidad de generar investigación, desarrollo y crecimiento competitivo. 


\section{Introducción}

En el proceso educomunicativo entran en juego diferente y diversas formas de expresión desde las del docente, los estudiantes, sin dejar de considerar las voces y posturas de los discursos de autores, instituciones y organismos y, por supuesto las expresiones institucionales y organizativas. Cada una de estas expresiones, ideas, experiencias, conocimientos, discursos, políticas, normas se filtran al aula al ser referentes en las lecturas y actividades llevadas a cabo en las diferentes materias de las carreras de ciencias de la educación y letras españolas. Pero, además, la percepción y las experiencias juveniles fuera del ámbito macro y micro institucional educativo se nutren de las relaciones $\mathrm{e}$ interacciones en que vive $\mathrm{y}$ conviven las y los estudiantes universitarios.

Por lo anterior, se considera que el significado psicológico (SP), compuesto de elementos afectivos y de conocimientos, son las representaciones subjetivas de la cultura y del entorno y, en este contexto, las redes semánticas naturales (RSN) posibilitan explorar, identificar, comparar y describir un concepto, en este caso formación profesional, a partir de las palabras definidoras (PD) individuales conjuntadas en las perspectivas de género y la carrera estudiada, donde se analizan las coincidencias y diferencias de las relaciones psicológicas y expresiones semánticas $\mathrm{y}$, en su caso, las PD concurrentes entre los grupos estudiados.

Como explica Valdez (2010), la Técnica de las Redes Semánticas Naturales (RSN), se ha ido consolidando como técnica confiable en el estudio del cómo el ser humano organiza la información y su efecto en la conducta humana. Sin dejar de lado las controversias, el estudio de la memoria implica la forma en que las personas retienen la información; sin embargo, la memoria no es un hecho aislado y específico, sino como proceso activo de construcción y reconstrucción de la información (pp. 15-32)

Los estudios sobre la memoria mostraron que la memoria a largo plazo (MLP) es la más compleja y, por sus características, es la más difícil de estudiar; en 1972, Tulving (como se citó en Valdez, 2010) distingue dos tipos más de memoria que se ubican en la MLP, la memoria episódica y la memoria semántica (pp. 33-38).
En el primer caso, "recibe y almacena información acerca de datos temporales, de episodios y eventos, y de las relaciones espaciotemporales, que se dan entre estos eventos" (p.39) Mientras que, en la memoria semántica:

Se dice que esta es necesaria para el uso del lenguaje. Es un tesoro mental, que organiza el conocimiento que una persona posee acerca de las palabras y otros símbolos verbales, sus significados y sus referentes, acerca de las relaciones entre ellos y acerca de las reglas, fórmulas y algoritmos para la manipulación de estos símbolos, conceptos y relaciones (Tulving, 1972 como se citó en Valdez, 2010, p.39).

\subsection{Objeto formal}

Cuando se alude a formación profesional (FP), es común que se relacionen dos de los principales ámbitos que están en juego: la educación y el mercado laboral por el desafío actual que significa la incorporación de los futuros egresados al sector productivo.

Ciertamente, la FP se relaciona desde el currículo, la complejidad, lo históricosociocultural, las expectativas estudiantiles e inclusive desde las propuestas de instituciones y organismos nacionales e internacionales; sin embargo, la FP en sí misma está cargada de subjetividades individuales, grupales y sociales. De esta forma, "el significado psicológico constituye un código de información relativo a un objeto en particular, ante el cual se presenta un comportamiento bastante específico y que también depende en gran medida de su base social o consensual que tenga". (Valdez, 2010, p.13)

Se propone explorar, leer y analizar desde una perspectiva cualitativa, las relaciones entre los estudiantes y el significado psicológico de la FP desde las diferencias y coincidencias que se observan en los significados psicológicos (SP), entre hombres y mujeres y sus carreras de origen.

Para analizar los SP de formación profesional, se recurre a las Redes Semánticas Naturales (RSN) propuesta por Figueroa (1981) y de Valdez (2010), ya que posibilita explorar las ideas, los imaginarios, las percepciones, en este caso de la PE formación professional. 
Así mismo, las explicaciones de la selección y estructuración de los significados, a través de la memoria semántica, permite conocer lo que los estudiantes saben, las asociaciones, las interacciones y las relaciones que le dan sentido a su realidad.

\subsubsection{Interrogante y objetivos}

En este sentido se cuestiona ¿qué significados psicológicos de la palabra estímulo formación profesional tienen los estudiantes de las licenciaturas en Ciencias de la Educación y la licenciatura en Letras Españolas?

El objetivo general que se desprende de la interrogante es el analizar las diferencias de significados psicológicos de formación profesional en mujeres y hombres a partir de la carrera estudiada.

Y los objetivos específicos, refieren a Describir el tipo de expresiones semánticas sustantivos, verbos, adverbios-utilizado en las palabras definidoras por ambos géneros y carreras; Analizar las palabras definidoras coincidentes y diferentes desde la perspectiva de género y carrera y, Comparar las palabras definidoras entre ambos géneros desde la perspectiva de carrera.

\subsubsection{Hipótesis}

Se parte del supuesto de que las representaciones psicológicas de la palabra estímulo formación profesional, está mediada por las representaciones e imaginarios de los diferentes espacios donde estos viven y conviven los estudiantes; además, de acuerdo con el género femenino o masculino, las palabras definidoras reflejarán sus experiencias y expectativas sobre la formación profesional, condicionadas por la carrera que están cursando.

En el apartado 2 Formación Profesional: aproximaciones, se aborda lo relacionado con las propuestas autorales, de instituciones y de organismos internacionales que sobre formación profesional explican y sus posturas sobre la misma. En el apartado 3 Metodología, se explica la tipología y diseño de investigación; misma que al recurrir a la técnica de Redes Semánticas Naturales (RSN), es de diseño cualitativo, transversal y que permite el análisis de los resultados desde una perspectiva descriptiva y comparativa y recurriendo al muestreo por conveniencia.
Lo que alude a los resultados, estos se podrán revisar en el apartado 4, donde se presentan los diferentes análisis a los que se recurrió al explorar y comparar las palabras definidoras (PD) de los estudiantes participantes. Finalmente en el apartado 5, se presentan las conclusiones generales del estudio.

\section{Formación profesional: aproximaciones}

\subsection{Educación y formación profesional: sus relaciones}

En México la educación superior, es impartida posterior a la terminación de la preparatoria o bachillerato. En el ámbito de la educación formal, para la SEP (2019) la educación superior es entendida como "una educación de calidad que permita la formación de profesionistas competitivos y comprometidos con el desarrollo regional y nacional, para contribuir a la edificación de una sociedad más justa”.

En la educación superior existen los niveles: Técnico Superior Universitario, Normal, Licenciatura y Postgrado tanto públicos como privados. Para efectos de este trabajo, se enfoca en la descripción del nivel licenciatura la cual son "Conocimientos avanzados en un campo de trabajo o estudio que requiera una comprensión crítica de teorías y principios" (SEP, 2017).

En este contexto, la educación formal son aquellos procesos de aprendizaje-enseñanza intencionados que se desarrollan al interior de instituciones públicas o privadas reconocidas por el Estado; es decir la educación, presencial o semi presencial, escolarizada o a distancia será aquella, normativamente hablando, en la que se imparten contenidos con una estructura lógica, un perfil de egreso delimitado en un espacio y tiempo previamente determinados en el diseño curricular y que certifica los aprendizajes y conocimientos adquiridos por los sujetos. Las certificaciones reflejadas en títulos pueden garantizar o no la inserción de los egresados al sector productivo. (UIS,2013)

Las universidades desempeñan el rol de formadores profesionales donde se desarrollan capacidades y habilidades que generalmente son demandadas por los empleadores. 
$\mathrm{Al}$ respecto en el 2016, posterior a un estudio realizado por el Banco Mundial, se enlistó una serie de competencias cognitivas habilidades mentales para comprender, razonar, adquirir nuevos conocimientos- y socioemocionales -resolución de problemas, pensamiento crítico y complejo- que "se relacionan con diferenciales positivos de salario y empleo para quienes las poseen". (OIT, 2017)

La relación entre instituciones de educación superior y formación profesional presenta diferentes matices, pero al mismo tiempo encuentran relaciones significativas que, como todo proceso humano, es complejo, multidimensional, holístico, y en estrecha relación y dialogicidad con su entorno.

Los autores consultados coinciden que la formación profesional en espacios universitarios, en su estrecha relación con el currículum, es permanente y progresiva, en constante interacción con los ambientes socioculturales, políticos, económicos e históricos donde este proceso se desarrolla. (Valera, 2010; Echeverry y López (2010), Llerena (2015).

En el acercamiento que se realiza entre la formación profesional basada en competencias como propuesta de integralidad de los egresados, se menciona que la formación profesional es donde se presenta la construcción de significados y sentidos entre los involucrados en el proceso de aprendizaje-enseñanza. (Valera, 2010)

En ese mismo sentido al abordar el currículo desde la complejidad, se indica que las instituciones de educación superior al incorporar la trama sociocultural, "retroalimentan prácticas, producciones, discursos e imaginarios socioculturales." (Echeberry y López, 2010); además que en la construcción identitaria se entretejen las identidades de la institución de formación, pero también las experiencias y trayectos personales que, finalmente, construyen al individuo profesional. (Navarrete, 2013)
En Llerena (2015) la formación profesional, desde la perspectiva de la complejidad e histórico-cultural, se considera que la educación superior y el gobierno deben de responder a las demandas de los estudiantes que ven en la formación profesional, su propio desarrollo personal y profesional.

Respecto a los planteamientos de los organismos internacionales sobre la educación superior (ES), formación profesional ${ }^{\mathbf{1}}(\mathrm{FP})$ y el mercado laboral (ML), la FP refiere a, "las actividades que tienden a proporcionar la capacidad práctica, el saber y las actitudes necesarias para el trabajo en una ocupación o grupo de ocupaciones de cualquier rama de actividad económica" (OIT, 2017; p. 7).

Para este organismo, la FP es donde se articulan las expectativas de los egresados para tener un trabajo decente, las políticas de desarrollo productivo y las políticas laborales. (OIT, 2017; p. 14)

La ES y su relevancia con el ML, estriba en que la primera, es el espacio privilegiado para la formación profesional, donde los estudiantes adquieren conocimientos y desarrollan las capacidades que los posibilita la inserción en diferentes ocupaciones laborales. (OCDE, 2019)

Sin embargo, la educación universitaria todavía no alcanza a dilucidar las peculiaridades que debe de reunir la FP para satisfacer las necesidades y problemáticas sociales.

\subsection{Formación profesional: expectativas universitarias}

La visión de los jóvenes sobre los temas que se abordan en este artículo es ineludible, ya que conocer los significados que un estudiante asigna a su comportamiento puede ser diametralmente opuesto a los que un investigador, un maestro o sus propios compañeros atribuyen al mismo; las interpretaciones difieren por el género, sus propósitos, experiencias e intereses.

En Chaves (2010) (como se cita en Borobia (2012) se señala que:

\footnotetext{
${ }^{1}$ En el documento de la OIT (2017) se aclara que la "formación profesional" puede ser referida indistintamente como "como capacitación laboral, formación laboral o formación para el trabajo" (p. 7) 
El efecto que produce en la constitución de cada generación la desigual apropiación de los medios de producción material y simbólica. Afirma que así resulta la imposibilidad de pensar en una juventud, ya que sus experiencias de vida, sus manifestaciones y sus identificaciones configuran diversas juventudes. Se construyen las identidades frente a los otros $y$ se generan identificaciones grupales $o$ colectivas. (p.702)

Para hablar de estas identificaciones, Chaves (2010) elige la noción de complicidad que la utiliza "para nombrar el lazo que une, agrupa, da sentido de comunidad o comunión a los que se consideran semejantes o iguales". (Como se citó en Borobia, 2012)

En relación con lo anterior, en una compilación de estudios sobre expectativas de los estudiantes realizados por Pichardo, García, De la Fuente y Justicia (2007), se destaca que las expectativas juveniles presentan estabilidad aún antes de llegar a la universidad, sobre todo en las cuestiones académicas; sin embargo, existe un desfase entre expectativas y experiencias debido al desconocimiento de vida universitaria, esto relacionado con las interacciones con los maestros (Como se cita en Sibrian, 2017, p.27). De acuerdo con resultados del estudio realizado por Sibrian (2017), las expectativas de los estudiantes universitarios giran en tres ejes principalmente:

1. Preparación para el mundo laboral y social.

2. Esperan que sus maestros presenten competencias docentes para que les ayude en el desarrollo de sus propias competencias, aprendizaje de nuevos conocimientos y un acompañamiento cercano

3. Y como parte de sus autoexpectativas, se comprometen a dar lo mejor de sí, "ser una persona responsable $\mathrm{y}$ obediente", aprovechar las oportunidades, apoyar a su familia y para dar lo mejor de sí en el empleo.

En el imaginario juvenil de esta población, la educación y FP son el puente para insertarse social y laboralmente de manera exitosa y comprometida. (Sibrian, 2017; pp.3032),
Sin embargo, lo que refiere a los universitarios, FP y el mercado laboral, Mancini (2014) alude al deterioro de las condiciones laborales que son resultado de la desregulación y flexibilidad laboral; característica que tienen alta presencia en los países de América Latina debido a la globalización y la acumulación del capital. (como se cita en Silva y Rodríguez, 2017; p. 95)

En este contexto, en Silva y Rodríguez (2017; pp. 97-98) se distinguen tres posturas con respecto al empleo juvenil aún y a pesar de la precariedad laboral que se cierne sobre este sector:

1. Estudiantes universitarios encuentran motivos para trabajar. Llevan a cabo su primer acercamiento hacia el ambiente laboral, partiendo de las necesidades económicas, el sentimiento de autonomía y autosuficiencia que es generado al recibir una remuneración económica, la adquisición de experiencia que permita el enriquecimiento de su currículum, o en algunos casos, combinar la actividad académica con el trabajo a fin de atender las exigencias de los empleadores para la obtención del grado en su desempeño profesional, así como para crear una vinculación de sus estudios con la vida profesional. (Beduwe y Giret, 2004; Guzmán 2004 y, Planas y Enciso, 2014, como se cita en Silva y Rodríguez, 2017)

2. Estudiantes que encuentran motivos para no realizar una temprana vinculación con el sector de la profesionalización, el tiempo que tienen no les es suficiente para realizar ambas actividades, las demandas institucionales con relación al tiempo de aprovechamiento académico truncan las oportunidades de obtener un trabajo, y en algunos casos, la carrera les proporciona la suficiente experiencia, por lo que no es indispensable el acercamiento al ambiente laboral. (Planas y Enciso, 2014, como se cita en Silva y Rodríguez, 2017) 
3. La aspiración social a la educación universitaria es una motivación que no sólo surge de manera intrínseca, sino que es fomentada fuertemente a través de los vínculos familiares. (Aramburu y Fernández, 1999, como se cita en Silva y Rodríguez, 2017)

Cual sea las condiciones reales a las que se enfrenta los estudiantes, tanto mujeres como hombres aún le apuestan a ingresar a la universidad, aun cuando las condiciones para el ingreso al sector productivo no son muy halagüeñas, como ya se ha referido anteriormente.

Llegar al término de la FP requiere de un gran esfuerzo y compromiso por parte de los estudiantes, que involucra no solo lo propio sino cumplir satisfactoriamente las normas y reglamentos académicos e institucionales que requieren y exigen de los individuos, madurez y habilidades sociales. Sin embargo, comentan Espinoza, Mustre, Nieto, Kent y Valle (2011):

Existen grandes diferencias entre las demandas de los estudiantes de nuevo ingreso, los campos de trabajos considerados emergentes, las capacidades reales del aparato productivo y las necesidades derivadas de las problemáticas sociales, ambientales, políticas y económicas de nuestro país, tanto por los rezagos estructurales históricos, como por su inserción en las nuevas circunstancias de su entorno. (p.43)

De Ibarrola (2010, p. 36) consigna el hecho de que, en lo individual, en la familia y en grupos sociales, permea la idea de que la escolaridad es sinónimo de mejores empleos y mejores condiciones de vida. Comenta De Ibarrola, "difícilmente se encontrarán grupos familiares que no deseen una escolaridad para sus hijos, y de preferencia, un acceso a la educación superior, aunque la experiencia de muchos jóvenes empiece a cuestionar la generalidad de los resultados anhelados".

\subsection{Educación y perspectiva de género}

El debate sobre la perspectiva de género no ha estado exento de controversias; se ha usado y se sigue utilizando en algunas posturas políticoideológicas como abanderamiento de las causas de las mujeres, como si el hombre no formara parte del género, no sufrieran violencia, discriminación y exclusión.
$\mathrm{Al}$ respecto, Palomar (2016) comenta en el ámbito académico el género:

Suele sustancializarse, creando el efecto de que su significado es pleno, único, claro y transparente cuando se usa como sinónimo de mujeres o de desigualdad social, sin que por lo general se haga precisión conceptual alguna; el disimulo académico respecto a la confusión semántica del género puede verse también en el trato indistinto del término como concepto, como categoría, como objeto, como adjetivo, como sistema, como esquema, como estructura (pp.42-43).

En estas vicisitudes el término género comenzó a utilizarse como lo políticamente correcto, aunque al mismo tiempo se manifestaba la confusión entre lo que se dice y quien lo dice. Al respecto, Yébenes (2014), señala que:

En el registro simbólico, el sujeto comparte con otros su mundo de significados fundamentales a través de "una fantasía subjetivamente objetiva", creada a partir de relaciones sociales y que es verdadera en tanto que ofrece una identidad reconocible en el mundo social. El exceso significativo tiene que ver con que, al estar en el mundo de los significantes propio de lo social, el significante está siempre sujeto a efectos retroactivos a través de los cuales pueden serle atribuidas nuevas maneras de significar; y en tanto tales, los significados conforman un sistema que, lejos de ser estable, está expuesto a la indeterminación de su apropiación en el ámbito social (como se cita en Palomar, 2016; p. 47)

En el portal del Gobierno de México, se indica que la perspectiva de género favorece la comprensión de la vida y las relaciones de hombres y mujeres; además posibilita mirar con otra perspectiva las relaciones humanas, reelaborar contenidos que abran las posibilidades de eliminar estereotipos y desequilibrios. Para solucionar estos desequilibrios, se propone:

Redistribución equitativa de las actividades entre los sexos (en las esferas de lo público y privado). Justa valoración de los distintos trabajos que realizan mujeres y hombres, especialmente en lo referente a la crianza de las hijas e hijos, el cuidado de los enfermos y las tareas domésticas. 
Modificación de las estructuras sociales, los mecanismos, las reglas, prácticas y valores que reproducen la desigualdad. El fortalecimiento del poder de gestión y decisión de las mujeres. (Comisión Nacional para Prevenir y Erradicar la Violencia Contra las Mujeres, 2018)

Pero más allá de lo políticamente correcto, de las ambigüedades, de las confusiones lo cierto es que las mujeres y los hombres piensan, sienten, se comportan, viven la vida de diferente manera. Sus experiencias están marcadas, por lo individual y también por las interacciones familiares, escolares, entre amigos, compañeros; la información que se recibe, lee, se consulta también impacta en las identidades individuales y grupales.

\section{Metodología \\ 3.1 Tipo de investigación}

El estudio Formación profesional. Significados psicológicos desde la perspectiva de género es cualitativa y transversal; así mismo, la investigación explora, describe y compara los significados de la palabra estímulo-definidoras de formación profesional desde la perspectiva de género y carrera universitaria.

\section{2 Ámbito de estudio}

El estudio se realizó y desarrolló durante el semestre agosto-diciembre; con la participación de la población de $1^{\circ}, 3^{\circ}, 5^{\circ}$ y $7^{\circ}$ semestre de las licenciaturas en Ciencias de la Educación (LCE) y Letras Españolas (LLE) de la Facultad de Ciencia, Educación y Humanidades (FCEyH) de la Universidad Autónoma de Coahuila (UAdeC). La FCEyH se encuentra ubicada en la Unidad Camporredondo de Saltillo, Coahuila.

\subsection{Muestra}

La población en agosto-diciembre de la LCE era de 143 estudiantes y 77 estudiantes de la LLE. Las mujeres y hombres participantes fueron voluntarios lo que indica una muestra no probabilística, por conveniencia o disponibilidad.

\subsection{Variables}

Acorde con la técnica de las $\mathrm{RSN}$ se recurre a la palabra estímulo (PE) formación profesional y experiencia.
Sin embargo, en este artículo solamente se analiza y presentan los resultados de la primera PE. Además de lo anterior, se recurre a variables demográficas, tales como género y edad, estas enmarcan las PD resultantes de las representaciones psicológicas y expresiones semánticas de los estudiantes. Así mismo, se incluyen variables socioeducativas, tales como licenciatura y si los estudiantes trabajan o no.

\subsection{Técnica de investigación}

Las Redes Semánticas Naturales (RSN) se considera un método de recolección de información que es utilizada tanto en investigaciones cuantitativas como cualitativas; es Figueroa, González y Solís (1981) quienes proponen trabajar con las estructuras generadas por humanos [naturales] y no con las redes elaboradas por computadora. (Reyes, 1993, p. 86) Valdez (2000) considera que estás [estructuras humanas] tienen su origen en los trabajos realizados por Tulving (1972) respecto a dos tipos de memoria, "la memoria episódica que recibe, almacena información de datos temporales (...) y la memoria semántica, que organiza el conocimiento acerca de las palabras y otros signos verbales, sus significados, sus referencias y las relaciones entre ellos". (como se cita en Álvarez-Gayou, 2013; p. 166)

En dos grupos de experimentación aplicando los mismos estímulos, pero recurriendo a las técnicas de asociaciones libres y las RSN (Figueroa, González y Solís, 1981) los resultados mostraron que ambas técnicas son "productos de procesos diferentes". Puede entenderse a las redes semánticas, como la recuperación de un conjunto de palabras precisamente a través de la memoria, dentro de procesos reconstructivos y no solamente asociativos, al estar determinada por las clases y propiedades de los elementos que la integran (Valdez-Medina, 2010, pp.60-62)

Además, se han realizado estudios que validan las RSN como técnica para evaluar el significado psicológico (SP) de cualquier concepto. Como ejemplo, es los realizado por Valdez-Medina (2010, pp. 87-91) con los conceptos de rico/pobre, donde su hipótesis alude a que "al usar cualquier otro instrumento que evalúe el significado, se encontrarían resultados iguales o muy similares a los encontrados mediante el uso de las redes semánticas naturales". 
Los resultados mostraron que a través del análisis factorial se logran distinguir los SP encontrados con anterioridad en las RSN para las dos PE y los estímulos distractores incorporados al instrumento no encontraron significatividad; así mismo que los niveles de correlación (Sperman) entre las dos técnicas, fueron significativos más allá del .01.

La técnica de las RSN, posibilita obtener información de forma veraz, ya que son los propios sujetos los que proporcionan $y$ jerarquizan las PD, dando como resultado la distancia semántica que indica que los conceptos obtenidos no serán igual de importantes para definir la PE.

Además, el significado psicológico de PE evidencia que la cultura de su entorno permea en las ideas, en los constructos o conceptos, posibilitando la descripción y diferenciación de un concepto a partir de los datos generados por un grupo de personas.

En la propuesta de las RSN, los individuos participantes son los que en libertad seleccionan, jerarquizan, generan los conceptos que crean que están relacionados o definan los significados de las PE para que el investigador sea el que, a través de un proceso validado de acopio y proceso de las palabras definidoras (PD), explore los adjetivos, sustantivos y/o verbos para identificar las representaciones de la PE.

\section{Resultados}

\subsection{Descripción sociodemográfica de la población por género y carrera}

De los 36 estudiantes de la carrera de Ciencias de la Educación (LCE), 28 son mujeres y sus edades mostraron concentración entre los 18 a 21 años. Existe un equilibrio entre las mujeres que sí y no trabajan. En la Tabla 1, se observa que la mayoría continuará estudiando el nivel de maestría.

\begin{tabular}{|c|c|c|c|c|c|c|c|}
\hline Edad & Fr & Trabaja & $\mathrm{Fr}$ & $\begin{array}{l}\text { Continuar } \\
\text { Estudiando }\end{array}$ & Fr & $\begin{array}{l}\text { Nivel de } \\
\text { Estudio }\end{array}$ & Fr \\
\hline $18-19$ & 9 & $\mathrm{Si}$ & 12 & $\mathrm{Si}$ & 25 & Maestría & 17 \\
\hline $20-21$ & 8 & No & 16 & No & 1 & Postgrado & 6 \\
\hline $22-23$ & 6 & & & & & Fisioterapia & 2 \\
\hline $24-25$ & 2 & & & & & Diplomado & 1 \\
\hline $28-29$ & 2 & & & & & & \\
\hline
\end{tabular}

Tabla 1 Descripción demográfica LCE: mujeres Fuente: Elaboración Propia 2020
En la Tabla 2, se muestra que en la carrera de Letras Españolas (LLE) 17 son mujeres y las edades entre los 17 y 23 años existe un equilibrio entre estos. La mayoría de las mujeres no trabaja y con misma frecuencia indican continuar estudiando maestría. Se observa y constata coincidencia entre no trabajar y sí continuar estudiando.

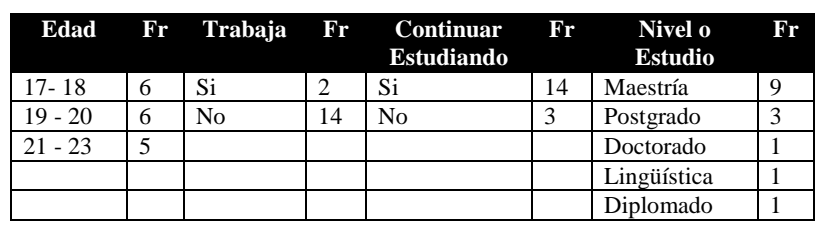

Tabla 1 Descripción demográfica LLE: mujeres Fuente: Elaboración Propia, 2020

En lo que refiere a la población varonil, en el caso de LCE, se encuentra una proporción similar entre las edades de 17 a 23 años. La mayoría no trabaja, pero seis (6) indicaron continuar sus estudios en el siguiente nivel escolar. Al igual que sus compañeras de la carrera de LLE, se encuentran similitudes entre continuar sus estudios y no estar trabajando.

\begin{tabular}{|c|c|c|c|c|c|c|c|}
\hline Edad & Fr & Trabaja & Fr & $\begin{array}{l}\text { Continuar } \\
\text { estudiando }\end{array}$ & Fr & $\begin{array}{l}\text { Nivel o } \\
\text { estudio }\end{array}$ & $\mathrm{Fr}$ \\
\hline $20-21$ & 4 & No & 7 & $\mathrm{Si}$ & 6 & Maestría & 4 \\
\hline $17-18$ & 2 & $\mathrm{Si}$ & 1 & No & 2 & Doctorado & 2 \\
\hline $22-23$ & 2 & & & & & Teología & 1 \\
\hline
\end{tabular}

Tabla 2 Descripción demográfica LCE: hombres Fuente: Elaboración Propia, 2020

Los cuatro (4) hombres de LLE, se evidencia un equilibrio entre las diferentes variables analizadas. Las edades están entre los 19 y 21 años e indicaron tanto trabajan como no; dos (2) indican continuar estudiando lingüística y maestría.

\subsection{Palabra estímulo y definidoras por género y carrera}

Para encontrar las PD de la PE, formación profesional se aplicó el instrumento a 57 estudiantes voluntarios de las carreras de LCE y LLE. Se trabajó con la propuesta original de las Redes Semánticas de Figueroa, González y Solís (1981), citado en Valdez Medina (2010); Reyes (1993) y Álvarez-Gayou (2003).

El valor J es de 300 palabras; este mismo valor en los estudiantes de la LLE, es de 166 palabras. 
Lo que refiere al peso semántico (Valor $\mathrm{M})$, se reducen las PD a través del proceso de eliminación del singular al plural o viceversa dependiendo del peso/jerarquía asignada por los estudiantes involucrados. De los procedimientos anteriores, se obtuvieron 15 PD del conjunto SAM desde la perspectiva de género y carrera.

\subsubsection{Palabras definidoras por género: sustantivos, verbos y/o adjetivos}

Lo que refiere a las mujeres de LCE se obtuvo un total de 106 PD y 81 de LLE. Las mujeres de ambas carreras para representar el significado psicológico de formación profesional utilizaron 179 sustantivos y utilizaron tres verbos (i.e. mejorar) y tres participios (i.e. egresado); mientras que las mujeres de la LLE recurrieron a un verbo y un participio (i.e. empleado).

Las respuestas de los hombres de la LCE y LLE se obtuvieron 47 y $24 \quad$ PD respectivamente. Los hombres LCE y LLE recurrieron a 69 sustantivos; por su parte, los varones de LCE utilizaron un participio (i.e. doctorado) y los de LLE utilizaron tres verbos (i.e. formar).

En ambos casos, se identifica que mayormente el léxico de ambos géneros y carreras recurren a la sustantivación, más que a la acción.

\subsubsection{Coincidencias y diferencias de PD por género y carrera}

En la Tabla 4, se identifican los conceptos que resulta en un $46 \%$ de coincidencia de las mujeres de ambas licenciaturas y un $53.33 \%$ de diferencia de las PD [Representado con el superíndice ${ }^{a}$ ]. El análisis indica que, para las mujeres de ambas carreras, la formación profesional está representada desde la perspectiva institucionalizada de los estudios y el conocimiento, además ellas consideran la temporalidad como finalización de una etapa de estudios donde se adquiere la práctica vinculada a la posterior incursión laboral.

\begin{tabular}{|c|c|c|c|}
\hline \multicolumn{4}{|c|}{ Mujeres } \\
\hline LCE & VMT & LLE & VMT \\
\hline Educación & 58 & Trabajo $^{\text {ad }}$ & 33 \\
\hline Trabajo $^{\text {ac }}$ & 57 & Estudio $^{\mathbf{a}}$ & 31 \\
\hline Estudio $^{\mathbf{a}}$ & 53 & Dedicación & 26 \\
\hline Maestro (s) a c & 48 & Esfuerzo & 24 \\
\hline Conocimiento $^{a}$ & 41 & Experiencia ${ }^{\text {a d }}$ & 19 \\
\hline Escuela $^{\mathbf{a}}$ & 35 & Confianza & 18 \\
\hline Aprendizaje & 33 & Maestro(s) ${ }^{\text {a d }}$ & 16 \\
\hline Experiencia $^{a}$ & 33 & Metas & 15 \\
\hline Valores & 28 & Alumnos & 14 \\
\hline Licenciatura & 25 & Calidad de Vida & 14 \\
\hline Desarrollo & 24 & Dinero $^{d}$ & 14 \\
\hline Habilidades & 22 & Tiempo $^{\mathbf{a}}$ & 14 \\
\hline Carrera & 21 & Conocimiento $^{\mathbf{a}}$ & 13 \\
\hline Tiempo $^{\mathbf{a}}$ & 21 & Escuela $^{\mathbf{a}}$ & 12 \\
\hline Práctica (s) ${ }^{\mathbf{c}}$ & 20 & Éxito & 12 \\
\hline
\end{tabular}

Tabla 4 Conjunto SAM: Mujeres

Fuente: Elaboración Propia, 2020

En Tabla 5, las PD muestran un $13.33 \%$ coincidencias y un $86.66 \%$ de diferencias entre los hombres de ambas careras [Identificadas con el superíndice ${ }^{b}$ ]. Se evidencia que las representaciones de los varones están vinculadas a la dimensión micro institucional y a las relaciones que se establecen con los docentes.

\begin{tabular}{|c|c|c|c|}
\hline \multicolumn{4}{|c|}{ Hombres } \\
\hline LCE & VMT & $\mathbf{L L E}$ & VMT \\
\hline Trabajo $^{\mathbf{b c}}$ & 28 & Maestro (s) & 14 \\
\hline Autonomía & 17 & Trabajo $\mathbf{b d}$ & 14 \\
\hline Expectativa & 14 & Asesores & 11 \\
\hline Actitud & 12 & Amigos & 10 \\
\hline Académico & 10 & Dinero $^{d}$ & 10 \\
\hline Demanda & 10 & Edad & 9 \\
\hline Liderazgo & 10 & Institución & 8 \\
\hline Maestro $(\mathrm{s})^{\mathbf{b} \mathbf{c}}$ & 10 & Secretarios & 8 \\
\hline Calidad & 9 & Experiencia $^{d}$ & 7 \\
\hline Enseñanza & 9 & Rector & 7 \\
\hline Prestigio & 9 & Aspiración & 6 \\
\hline Capacitación & 8 & Buscar & 6 \\
\hline Interés & 8 & Estudios & 6 \\
\hline Práctica $(\mathrm{s})^{\mathbf{c}}$ & 8 & Familia & 6 \\
\hline Resolución & 8 & Compañeros & 5 \\
\hline
\end{tabular}

Tabla 5 Conjunto SAM: Hombres Fuente: Elaboración Propia, 2020

Finalmente, dentro de este rubro, existe un $86.66 \%$ de diferencia entre los hombres y mujeres de LCE [Representado con el superíndice $^{\mathrm{c}}$ ] y un $73.33 \%$ entre ambos géneros de LLE [Simbolizado con el superíndice ${ }^{\mathrm{d}}$ ].

En lo que refiere a la muestra de LCE, las PD coincidentes connotan la importancia que para ellos tienen los docentes y las prácticas en su formación profesional, lo que ayuda en su futura inserción laboral. 
Lo que refiere al $26.67 \%$ de coincidencia entre mujeres y hombres de LLE [Superíndice ${ }^{\mathrm{d}}$ ], sugieren el involucramiento de los maestros en su formación y la necesaria experiencia que se requiere para iniciarse en el ámbito laboral, que reditúa en percepciones económicas por su trabajo.

Desde la perspectiva de género, las mujeres presentan PD coincidentes y diferentes casi en la misma proporción a diferencia de los varones y entre los compañeros de la misma carrera.

En las Tablas 4 y 5 se identifican que maestros y trabajo, son las palabras definidoras (PD) coincidentes en los cuatro grupos analizados.

Lo que evidencia la importancia de los docentes en su formación académica con perspectivas al trabajo.

\subsubsection{Representaciones psicológicas de formación profesional: género y carrera}

En la Tabla 4 se observa que las representaciones de la formación profesional de las mujeres de LCE tienden hacia el proceso de aprendizajeenseñanza (i.e. conocimiento, aprendizaje) y lo que este desarrolla y fomenta en su formación (i.e. aprendizaje, habilidades); dichas representaciones se enmarcan en un contexto institucional micro (i.e. escuela, estudio) y se evidencia una relación filial con los docentes. Además, consideran el involucramiento personal en ese proceso formativo (i.e. tiempo, desarrollo, carrera), incluidas las cuestiones éticas-morales. Se observa, además, que las representaciones femeninas están ancladas en su momento y espacio actual (i.e. tiempo, licenciatura) por tanto se encaminan hacia esta formación profesional que les permitirá incursionar en el sector productivo (i.e. experiencia, trabajo).

Mientras que las representaciones de las mujeres de LLE, connotan las implicaciones personales que para ellas tiene el estudiar una carrera (i.e. esfuerzo, dedicación), pero al igual que sus congéneres de LCE, las PD aluden a las cuestiones de índole institucional micro y formativa (i.e. proceso de aprendizajeenseñanza), sin dejar de considerar las relaciones filiales que se establecen con los maestros.
Así mismo, desde la perspectiva personal, las representaciones psicológicas de las mujeres de LLE, sugieren que al término de la formación profesional su propósito está encaminado a la inserción al sector productivo (i.e. metas), lo que puede conllevar a tener ganancias económicas para el mejoramiento de su vida actual (i.e. dinero, calidad de vida, éxito).

Lo que atañe a las representaciones psicológicas expresadas semánticamente por los varones de LCE (ver Tabla 5), las PD sobre formación profesional implican cuestiones intrínsecas (i.e. actitud, interés) que posibilitan alcanzar, por una parte, su independencia (i.e. interés, expectativas) y por otra el reconocimiento social (i.e. liderazgo, prestigio). Así mismo, la formación profesional involucra lo que es la formación en sí misma (i.e. calidad, práctica) y los valores (i.e. autonomía) dentro del microambiente institucional (i.e. académico, maestros); finalmente, los hombres de LCE consideran cuestiones relacionados al ámbito laboral, tales como la capacitación, la demanda y el trabajo. Todas estas representaciones lo llevan a expresar semánticamente, lo que es la formación profesional: trabajar y obtener ganancias.

Mientras que para los hombres de LLE, las representaciones psicológicas de formación profesional por una parte refieren a aspectos más íntimos-filiales (i.e. familia, amigos, compañeros) y por otra a la edad, cuestiones que no se han identificado en los otros grupos analizados. También en estas representaciones se identifican aspectos intrínsecos (i.e. buscar, aspiraciones) y aspectos a nivel macro y micro institucionales (i.e. rector, maestros).

\section{Conclusiones}

Más allá, de las posturas ideológicas que se abanderen en lo relacionado a género, los análisis indican que existe diferencias significativas entre hombres y mujeres; además de identificar que, entre las mujeres/hombres el hecho de ser de diferente carrera de origen (LCE/LLE) sus representaciones psicológicas presentan así mismo variaciones.

Lo anterior indica que la carga valoral, cultural, sus experiencias, sus interacciones intra y extra muros permea y se reflejan en las palabras definidoras $(\mathrm{PD})$ que se expresaron. 
En las representaciones psicológicas, los filtros que se utiliza, además del género, es la carrera de origen; ya que se observó que las mujeres de LCE tienden más al proceso de $\mathrm{AE}$ y al espacio-tiempo de su estudio; sin embargo, las mujeres de LCE, reflejan el esfuerzo que implica el estudio de su carrera.

Estos filtros de género y carrera, en los hombres de LCE aluden a cuestiones más prácticas y de índoles social; mientras que los varones de LCE, sus representaciones están más enfocadas a las relaciones filiales-familiares.

Finalmente, lo evidente es que en ambos géneros y carreras, las coincidencia aluden a las relaciones maestros y trabajo.

Sin lugar a dudas, los resultados son atrayentes y con posibilidades a seguir la huella de estos estudiantes para ver si habían cambios en sus representaciones, sin embargo, la pandemia del COVID-19, frustró estas ideas.

\section{Referencias}

Álvarez-Gayou, J. L. (2013). Cómo hacer investigación cualitativa. Fundamentos y metodología. México, México: Paidós.

Comisión Nacional para Prevenir y Erradicar la Violencia Contra las Mujeres. (22 de Noviembre de 2018). ¿Qué es la perspectiva de género y por qué es necesario implementarla? Recuperado el 28 de Octubre de 2019, de Portal del Gobierno de México: https://www.gob.mx/conavim/articulos/que-esla-perspectiva-de-genero-y-por-que-esnecesario-implementarla

De Ibarrola, M. (Octubre de 2010). Siete preguntas claves sobre las relaciones entre educación y el trabajo en México ¿Qué aporta la investigación educativa). Cuadernos de educación, XIII(8), 33-75. Recuperado el 10 de Noviembre de 2018, de https://revistas.unc.edu.ar/index.php/Cuadernos /article/view/796/751

Echeverri, G., \& López, B. (2010). El currículo universitario: una propuesta compleja. Recuperado el 5 de Febrero de 2019, de Universidad Pontificia Bolivariana. Centro de Recursos Documentales e Informáticos. Sala del CREDI de la OEI: https://www.oei.es/historico/oeivirt/salacredi/E cheverryBeatriz.pdf
Espinoza, E., Mustre, J., Nieto, L. M., Kent, R., \& Valle, R. (2011). Calidad educativa. Un concpeto multidimensional. En E. Esponoza, \& V. (. Rosario, Calidad educativa. Concepto, contexto y percepción del profesorado. Guadalajara, Jalisco, México: Editorial Universitaria.

IIEP. (2012). Educación formal. Glosario. Recuperado el 13 de Abril de 2018, de UNESCO; Instituto Internacional de Planeamiento de la Educación: https://learningportal.iiep.unesco.org/es/glossar y/educaci\%c3\%b3n-formal

INET. (s/f). Formación profesional. Recuperado el 8 de Febrero de 2018, de Instituto Nacional de Educación Tecnológica, Ministerio de Educación:

http://www.inet.edu.ar/index.php/niveleseducativos/formacion-profesional/

INMUJERES; INEGI. (Mayo de 2018). Mujeres y Hombres en México 2018. Recuperado el 20 de Febrero de 2019, de INMUJERES;INEGI: http://cedoc.inmujeres.gob.mx/documentos_do wnload/MHM_2018.pdf

Llerena, O. (Septiembre-Diciembre de 2015). El proceso de formación profesional desde un punto de vista complejo e histórico-cultural. Actualidades Investigativas en Educación, 15(3), 1-23. doi:http://dx.doi.org/10.15517/aie.v15i13.2104 1

Navarrete, Z. (Julio-septiembre de 2013). La universidad como espacio de Formación profesional y constructora de identidades. Recuperado el 10 de Diciembre de 2018, de Red de Revistas Científicas de América Latina y el Caribe (Redalyc): http://www.redalyc.org/articulo.oa? $\mathrm{id}=3733124$ 6003

OCDE. (2010). Habilidades y Competencias del siglo XXI para los aprendices del nuevo milenio en los países de la OCDE. Recuperado el 17 de Agosto de 2017, de Instituto de Tecnologías Educativas: http://www.ite.educacion.es/

OECD. (2019). El futuro de la educación en México. Promoviendo Calidad y Equidad. México: OECD. Recuperado el 25 de Septiembre de 2019, de https://www.oecd.org/centrodemexico/medios/e 1_futuro_de_la_educacion_en_mexico.pdf

YAÑEZ-FLORES, Sara Margarita, HERNÁNDEZ-CUETO, Jaquelina Lizet, SALINAS-AGUIRRE, María del Consuelo y VALDES-URIBE, Alejandra Guadalupe. Formación profesional. Significados psicológicos desde la perspectiva de género. Revista Teoría Educativa. 2020 
OECD. (2019). Higher Education in Mexico: Labour Market Relevance and Outcomes, . OECD, Higher Education. París: OECD. Recuperado el 23 de Julio de 2019, de https://doi.org/10.1787/9789264309432-en..

Palomar, C. (15 de Septiembre de 2016). Veinte años de pensar el género. Debate feminista(52), 34-49.

doi:http://dx.doi.org/10.1016/j.df.2016.09.002

Reyes, I. (1993). Las redes semánticas naturales su conceptualización y su utilización en la construcción de instrumentos. Revista de Psicología Social y Personalidad., IX(1), 81-97.

SEP. (2017). Educación sin fronteras. Recuperado el 12 de Febrero de 2019, de Secretaría de Educación Pública/SES: https://www.ses.sep.gob.mx/educacionsinfronte ras/

SEP. (2019). Educación Superior. Recuperado el 15 de Junio de 2019, de Secretaría de Educación Pública: https://educacionsuperior.sep.gob.mx/

Sibrian, L. (2017). Expectativas de la formación universitaria desde la perspectiva de los estudiantes. Diá-logos(19), 25-37. doi:https://doi.org/10.5377/dialogos.v.0i19.548 1

Silva, B., \& Rodríguez, A. (2017). Jóvenes Universitarios ante la Formación Profesional y la Precariedad Laboral. Población Y Desarrollo Argonautas $Y$ Caminante, 12, 95-104. doi:https://doi.org/10.5377/pdac.v12i0.3103

UIS. (2013). Clasificación Internacional Normalizada de la Educación. Biblioteca Digital UNESCO-UIS. Recuperado el 13 de Abril de 2018, de CINE: https://unesdoc.unesco.org/ark:/48223/pf00002 20782

Valdez-Medina, J. (2010). Las redes semánticas naturales, usos y aplicaciones en psicología social (4a ed.). México: Universidad Autónoma del Estado de México. 